\title{
El confinamiento como estrategia sanitaria y error de politica pública
}

\section{Lockdown as sanitary strategy and mistake of public policy}

\author{
Alvaro-J Idrovo ${ }^{1}$ (D)
}

Forma de citar: Idrovo AJ. El confinamiento como estrategia sanitaria y error de política pública. Salud UIS. $2021 ; 53:$ e21001. doi: https://doi.org/10.18273/saluduis.53.e:21001 (c) (1)

Desde el punto de vista sanitario, los confinamientos pueden ser de dos tipos: cuarentenas cuando involucra personas sanas, o aislamientos si son personas infectadas a las que se pide que se queden encerradas ${ }^{1}$. Si bien son medidas usadas desde tiempos antiquísimos, el paso de los años llevó a que se humanizaran un poco, pasando de confinamientos alejados del resto de la sociedad (incluida la familia), a hacerlas más llevaderas al estar en contacto con familiares. Desde luego esta humanización conllevó a un incremento del contagio dentro de los hogares y esto actualmente es visto como habitual en la pandemia de COVID.

La efectividad de la cuarentena era conocida, pero no había sido evaluada con métodos epidemiológicos modernos, lo cual se pudo hacer durante 2020 en varios lugares del mundo ${ }^{2-4}$, Esta evaluación sugiere que su uso adecuado debe ser en los primeros momentos de la epidemia para ganar tiempo y mejorar la respuesta sanitaria. Sin embargo, su uso ha sido excesivamente prolongado en regiones como América Latina (incluyendo Colombia) y esto genera repercusiones sobre los derechos civiles y la democracia ${ }^{5,6}$ que ya empiezan a evaluarse por los expertos en esas áreas del conocimiento. No obstante, su cumplimiento no siempre fue $100 \%$ efectivo dado que la pobreza, las desigualdades y otros determinantes sociales limitaron su potencial ${ }^{7}$, al no incorporar apoyos económicos entre los hogares con menos poder adquisitivo, y al cansancio y rebeldía cada vez más frecuentes después de pasar un tiempo en esta condición.

En Colombia además de estas formas de controlar la movilidad de las personas durante la pandemia, se han usado "innovadoras" formas para implementar el distanciamiento social como permitir la movilidad de solo una proporción de la población, en lo que comúnmente se conoce como "pico y cédula" y sus demás variantes, junto a los toques de queda.

El "pico y cédula" surgió durante 2020 teniendo similitudes con el "pico y placa" instaurado por primera vez en Bogotá en 1998 con el objetivo de disminuir el tráfico vehicular; éste, a su vez, era la expresión colombiana del "hoy no circula" que se implementó en 1989 en Ciudad de México para bajar la contaminación atmosférica al restringir la circulación de una proporción de vehículos ${ }^{8}$. La efectividad sanitaria de esta medida aún no es muy clara, o por lo menos las autoridades no la han hecho pública, y al parecer depende de quien deba verificar su cumplimiento que, en general, pasó a ser de comerciantes y vigilantes que sin duda ven la restricción como contraria a sus intereses.

Por su parte, el concepto de toque de queda proviene de la palabra en francés couvre-feu (cubrir y fuego en español), y se usó en el medioevo para definir la necesidad de apagar el fuego hacia las 8 o 9 pm y evitar los incendios; desde entonces se usa como término de control de la movilidad nocturna, principalmente. Algunos sugieren que el rey inglés Guillermo I "el conquistador" (1028-1087) empezó a usar el toque de queda como medida de represión política', lo que fue copiado a través de la historia en diversas regiones del mundo, y que durante la pandemia de COVID-19 ha sido aplicada en muchos países. Colombia, dada su historia de movimientos sociales y diferentes tipos de violencia, no ha sido ajena al uso de los toques de queda. Incluso, hay evidencia que muestra su efectividad para disminuir la ocurrencia de hechos violentos como los homicidios ${ }^{10}$. Dado que los microorganismos infecciosos

1. Universidad Industrial de Santander. Bucaramanga, Colombia

Correspondencia: Javier Idrovo. Dirección: Cra. 32 29-31, Bucaramanga. Teléfono: +57634 4000, ext. 3195. Correo electrónico: idrovoaj@uis.edu.co 
no incrementan su capacidad de transmisión en las horas de la noche, el uso de estas medidas responde a efectos no directamente relacionados con la pandemia, sino con hechos sociales relacionados que sí pueden hacer parte de la sindemia asociada al COVID-19; podemos señalar allí la violencia, el consumo de alcohol y los traumas, entre otros. La poca evidencia colombiana generada durante la pandemia apunta en ese sentido ${ }^{11}$, dejando de lado su efecto directo en el control del contagio.

Como se puede ver, los confinamientos siguen siendo las medidas sanitarias más efectivas para manejar la pandemia, pero constituyen una medida muy drástica porque detiene las actividades económicas y limita los derechos individuales. El "pico y cédula" es muy dependiente de quien la tenga que hacer cumplir y la capacidad gubernamental de verificarla, por lo que los resultados deben ser muy heterogéneos; de hecho, muchas noticias indican que solo cuando hay despliegue policial o casos donde hay sanciones o multas es que parece se cumple en mejor medida. El toque de queda responde más a objetivos fuera del campo de la salud, más policivos y de control social. Por ello, no es extraño que en los momentos críticos de la atención hospitalaria, cuando crece rápidamente el número de pacientes graves que desbordan las capacidades humanas y de infraestructura hospitalaria, así mismo, las asociaciones de profesionales de la salud han salido a pedir confinamientos estrictos. Por lo anterior, la pregunta que surge es ¿por qué no se previno que esto ocurriera mediante intervenciones menos restrictivas?, quizá aplicándolas durante un mayor lapso de tiempo, por ejemplo. La teoría basada en la organización en "burbujas sociales"12 sirve como base para implementar intervenciones en los ámbitos familiares, escolares y ocupacionales ${ }^{13}$. Ese camino intermedio, que prolonga en duración la pandemia pero permite que la economía siga viva, es complejo y exigente con los tomadores de decisiones. Pues exige alta creatividad y capacidad de gestión, lo cual no es habitual encontrar entre los gobernantes, y en lo que se ha visto en esta pandemia corresponde más a la excepción que la regla.

En consideración con lo anterior, que los profesionales de la salud ajenos a la salud pública entiendan la prevención y su importancia es difícil, y esto también ocurre entre los que toman las decisiones. Siempre ha resultado más fácil pensar en un medicamento de acción rápida, una "multi-píldora" o una cirugía que solucione el problema, como las formas favoritas de actuar en salud; es lo que se ha denominado "patologización", que incluso va más allá de la "medicalización"14. Por ello, muchos creen que la vacuna será la solución, cuando realmente ésta solo será un elemento más que se suma para ir superando exitosamente poco a poco la pandemia. Se necesita actuar preventivamente informando, comunicando, educando, empoderando, buscando cambiar comportamientos determinados por la historia, la cultura y la sociedad en su conjunto sin duda es difícil pero son mucho más efectivos a largo plazo. Las medidas de control social sin duda funcionan, mientras haya confianza o miedo en el gobierno y sus instituciones, pero son medidas que de repetirlas van perdiendo su efecto, y mucho más en un contexto de desigualdades sociales ${ }^{7}$. Además, no debe olvidarse que esas desigualdades han sido parte de la red causal involucrada en que se tenga alta ocurrencia de obesidad, diabetes, hipertensión y demás expresiones de multimorbilidad que complican la infección con SARS-CoV-2. Por ende, la "nueva realidad" para que funcione debería ser un compromiso gubernamental de apoyo y de la ciudadanía de cumplimiento, solidaridad y empatía.

Como se puede ver, la declaración de un confinamiento puede verse como una estrategia válida de salud poblacional cuando es al inicio de una epidemia, con el fin de ganar tiempo mientras se logra una mejor preparación para afrontarla, o como medida extrema cuando no quedan más opciones. En otros momentos, además, puede verse como error de política pública ${ }^{15}$; es una forma silenciosa, tácita, que indica que algo no se hizo bien. El escalonamiento en las restricciones, con indicadores públicos, transparentes y previamente conocidos debería ser la forma más adecuada de manejar los diferentes momentos de la pandemia, de manera que se evite el deterioro de la economía y el colapso hospitalario.

Mientras los tomadores de decisiones pueden llegar a concluir que lo que lleva al aumento de los casos nuevos y requerimientos de atención hospitalaria, especialmente en unidades de cuidado intensivo, es el incumplimiento social de protocolos y normas. Una visión más crítica y profunda de los hechos y conflictos sociales, permitiría entender que es un error mayor del tomador de decisión que no pudo corregir (ni siquiera de manera parcial y temporal) las desigualdades sociales.

Finalizo reiterando que la responsabilidad del manejo de la pandemia es principalmente de los gobernantes, porque gran parte de la ciudadanía no tiene las capacidades de afrontarla individualmente y requiere apoyos del Estado. 


\section{Referencias}

1. Centers for Disease Control and Prevention. Quarantine and isolation; 2021. https://www.cdc.gov/quarantine/ index.html

2. Girum T, Lentiro K, Geremew M, Migora B, Shewamare S. Global strategies and effectiveness for COVID-19 prevention through contact tracing, screening, quarantine, and isolation: a systematic review. Trop Med Health. 2020; 48:91. doi: https://doi.org/10.1186/s41182-020-00285-w

3. Nussbaumer-Streit B, Mayr V, Dobrescu AI, Chapman A, Persad E, Klerings I, et al.. Quarantine alone or in combination with other public health measures to control COVID-19: a rapid review. Cochrane Database Syst Rev. 2020; 9: CD013574. doi: http://10.1002/14651858.CD013574.pub2

4. Vali M, Mirahmadizadeh A, Maleki Z, Goudarzi F, Abedinzade A, Ghaem H. The impact of quarantine, isolation, and social distancing on COVID-19 prevention: a systematic review. J Health Sci Surveillance Sys. 2020; 8(4): 138-150.

5. Idrovo AJ. Long but unreal lockdowns in Latin America. Comment on Chen YT, Yen YF, Yu SH, Su EC. An examination on the transmission of COVID-19 and the effect of response strategies: A comparative analysis. Int. J. Environ. Res Public Health. 2020; 17, E5687. Int J Environ Res Public Health. 2020; 17(21): 8064. doi: http://10.3390/ijerph17218064

6. Bækgaard M, Christensen J, Madsen JK, Mikkelsen KS. Rallying around the flag in times of Covid-19: Societal lockdown and trust in democratic institutions. J Behav Public Admin 2020; 3(2): 1-12. doi: http://10.30636/ jbpa.32.172

7. Idrovo AJ. ¿La ciudadanía responsable de la pandemia? Un análisis desde los determinantes sociales. Salud UIS. 2020; 52(4): 362-364. doi: http://dx.doi.org/10.18273/revsal.v52n4-2020001

8. Carrillo PE, Malik AS, Yoo Y. Driving restrictions that work? Quito's Pico y Placa Program. Can J Econ. 2016; 49(4): 1536-1568. https://doi.org/10.1111/caje.12243

9. Marketos AK. The constitutionality of juvenile curfews. Juv Fam Court Journal 46(2): 17-30.

10. Burbano EJ, Villa E, Zafra MI ¿Los toques de queda para menores de edad disminuyen la tasa de homicidio? Evidencia de Cali (Colombia). Iberoam J Develop Stud 2020; 9(2): 174-206. doi: http://10.26754/ojs_ried/ ijds. 374.5

11. Ruiz-Pérez JI, Aparicio J. Análisis espacio-temporal del incumplimiento de normas legales sobre el confinamiento en Colombia por COVID-19. Revista Logos Ciencia \& Tecnología 2020;12(3):20-32. doi: https:// doi.org/10.22335/rlct.v12i3.1251

12. Idrovo AJ, Moreno-Montoya J, Pinzón-Flórez CE. Desempeño de las pruebas combinadas de IgM e IgG rápidas en la vigilancia ocupacional de COVID-19 en empresas colombianas. Biomédica. 2020; 40(Supl.2): 139-147. doi: https://doi.org/10.7705/biomedica.5829

13. Block P, Hoffman M, Raabe IJ, Dowd JB, Rahal C, Kashyap R, et al. Social network based distancing strategies to flatten the COVID-19 curve in a post-lockdown world. Nat Hum Behav. 2020; 4: 588-596. doi: https://doi. org/10.1038/s41562-020-0898-6

14. Sholl J. The muddle of medicalization: pathologizing or medicalizing? Theor Med Bioeth. 2017; 38: 265-2278. doi: http://10.1007/s11017-017-9414-z

15. Hernández LJ. El manejo político de la epidemiología. El Nuevo Siglo. https://www.elnuevosiglo.com.co/ articulos/01-11-2021-el-manejo-politico-de-la-epidemiologia?fbclid=IwAR2kkN_aJWWWPHpk1r_oKHoKc DxfhG_9eahGojSUzZbLpmHm07tcZpEcSUc 